Original Research Paper

\title{
Analysis and Simulation of the Dynamics of Nickel Anode Plate Handling Robot
}

\author{
Krich Richel Mpemissi Kombo, Guoping Li and Smart Valentine Mudzingwa \\ Mechanical Engineering, University of Jinan, Jinan, China
}

\author{
Article history \\ Received: 03-11-2021 \\ Revised: 23-11-2021 \\ Accepted: 10-12-2021 \\ Corresponding Author: \\ Guoping Li \\ Mechanical Engineering, \\ University of Jinan, Jinan, \\ China \\ Email: me_ligp@ujn.edu.cn
}

\begin{abstract}
As an integral part of the industrial automation system, a joint robot with artificial intelligence technology, virtual prototype technology and co-simulation technology has developed rapidly. In particular, the development of coordinated control technology makes the robot trajectory tracking control advance in the direction of higher precision and higher efficiency and makes the whole robot system has high fitting human nature, high universality, high scalability and so on. In this study, for the structural characteristics of the nickel anode plate handling robot, a three-dimensional model of the handling robot was drawn using SolidWorks 3D design software. The linkage coordinate system of the robot is established by the D-H method. The dynamics model of the nickel anode plate handling robot was established using the Lagrange method and dynamics analysis was carried out. The simulation model of the robot was constructed based on ADAMS simulation software and the dynamics simulation was carried out to obtain the force curves of the joints. It lays the foundation for further optimization of the structure design and control system design of the handling robot.
\end{abstract}

Keywords: Handling Robot, D-H Method, Dynamics Model, Lagrange Method, Dynamics Simulation

\section{Introduction}

Nickel has excellent mechanical properties and corrosion resistance, high thermal conductivity and conductivity, low gas content and low steam pressure. It is used in food processing equipment, salt refining equipment, mining and marine mining. It is also mainly used in electronic products, high-precision chemical instruments and other products; Handling materials is an important link in industrial production, especially when the materials are heavy and toxic. The workers handling materials will be inefficient and inefficient cause certain harm to the body. To effectively solve this phenomenon and save labor, handling robots came into being.

As important automation equipment in the modern manufacturing industry, the application of handling robots is becoming more and more widespread and the development of handling robots is becoming more and more rapid. In the research and design process of handling robots, the theory and application related to robot dynamics are getting more and more attention. Theoretical analysis of handling robot dynamics can improve the stability and accuracy of the control system. Meanwhile, dynamics simulation is beneficial to optimize the structural design of robots and evaluate and check the dynamics characteristics of existing robot structures $(\mathrm{Xu}$, 2007; Yi and Xue-Ping, 2009).

At present, the Newton-Euler method, Lagrangian method and Kane method are the main methods to study robot dynamics. For example, some researchers have applied ADAMS software to simulate the tracking trajectory in the space near the maximum load of the robot (Wang et al., 2017); some researchers have applied MATLAB software to analyze and simulate the dynamics of the simplified model (Liu and $\mathrm{Hu}, 2013$; Zeng and $\mathrm{Li}, 2014)$. The dynamics simulation of the robot by applying these methods verifies the rationality of the structural design or the correctness of the theoretical calculation. In this study, we take the nickel anode plate handling robot as the research object, establish the dynamics model of the handling robot and use ADAMS to plot the simulation curve to verify the correctness of the dynamics model. 


\section{Establishment of 3D Model of Nickel Anode Plate Handling Robot}

\section{Overall Solution Design}

The 3D model of this handling robot was created by SolidWorks, as shown in Fig. 1. It consists of a base, a large arm, a small arm, a wrist and a nickel anode plate fixture. In practice, the nickel-anode plate fixture is fixed at the end flange of the wrist, so the wrist end flange and the nickel-anode plate fixture are considered as a whole. The 6 degrees of freedom of the handling robot are $: 1$, rotation of the base 2, pitch of the large arm 3, the pitch of the small arm 4, rotation of the front of the small arm 5, pitch of the wrist 6 and rotation of the nickel anode plate fixture. The first three rotational joints of the handling robot determine the position of the nickel anode plate fixture and the last three rotational joints determine the attitude of the nickel anode plate fixture (Hu et al., 2016).

\section{Establishment of Connecting Rod Coordinate System}

In order to elaborate the connection between the end position of the nickel-anode plate fixture of the handling robot and each joint angle, the theoretical knowledge of D$\mathrm{H}$ was applied to construct the reference coordinate system of the handling robot (Zeng et al., 2010) and the results are shown in Fig. 2. Here, the origin $O_{6}$ of the tool coordinate system of this robot is defined as the end position point of the robot and the base is regarded as linkage $O_{0}$.

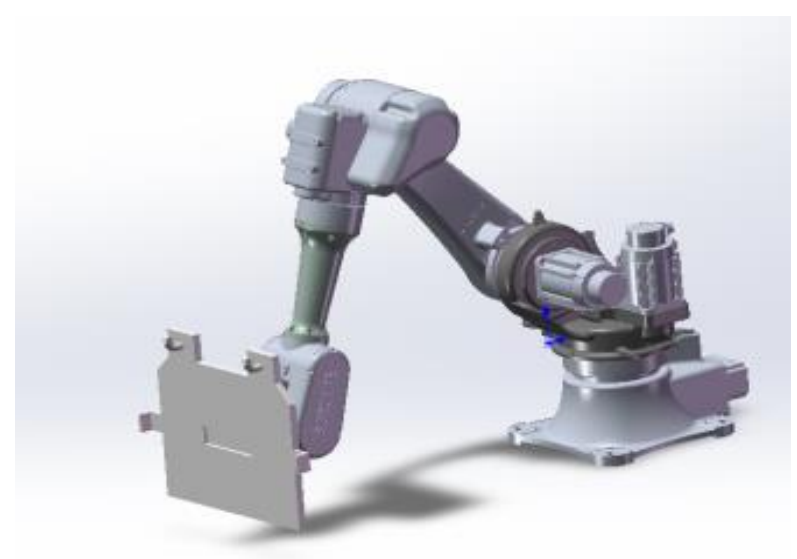

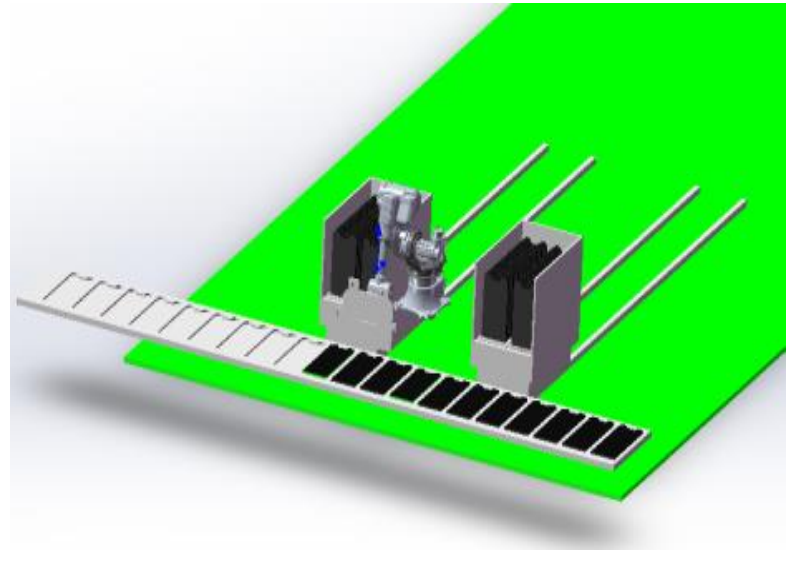

Fig. 1: Overall schematic diagram of nickel anode plate handling robot

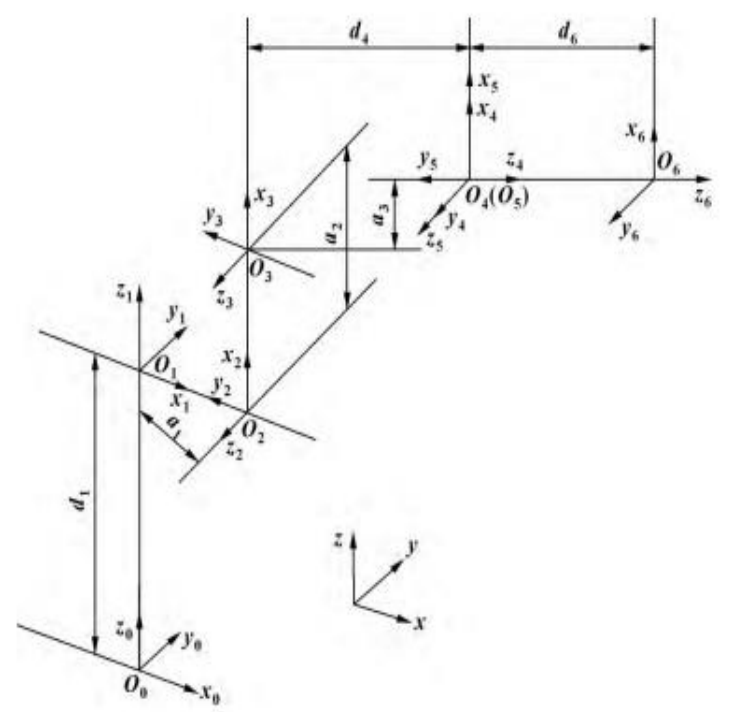

Fig. 2: Nickel Anode Plate Handling Robot Linkage coordinate system from the coordinate system of the linkage created in Fig. 2, the corresponding linkage parameters can be obtained, as shown in Table 1

Table 1: Nickel anode plate handling robot connecting rod parameters

\begin{tabular}{lllllll}
\hline Rod & $\begin{array}{l}\text { Joint corner } \\
\theta_{\mathrm{i}} /\left(^{\circ}\right)\end{array}$ & $\begin{array}{l}\text { Joint torsion } \\
\text { angle } \alpha_{\mathrm{i}-1} /\left(^{\circ}\right)\end{array}$ & $\begin{array}{l}\text { Rod length } \\
\mathrm{a}_{\mathrm{i}-1} / \mathrm{mm}\end{array}$ & $\begin{array}{l}\text { Rod distance } \\
\mathrm{d}_{\mathrm{i}} / \mathrm{mm}\end{array}$ & $\begin{array}{l}\text { Allowable range of } \\
\text { joint angle change/ }\end{array}$ & $\begin{array}{l}\left(^{\circ}\right) \\
\text { parameters } / \mathrm{mm}\end{array}$ \\
\hline 1 & $\theta_{1}$ & 0 & 0 & $\mathrm{~d}_{1}$ & \pm 180 & $\mathrm{~d}_{1}=563$ \\
2 & $\theta_{2}+90^{\circ}$ & 90 & $\mathrm{a}_{1}$ & 0 & $70 /-130$ & $\mathrm{a}_{1}=220$ \\
3 & $\theta_{3}$ & 0 & $\mathrm{a}_{2}$ & 0 & $175 /-80$ & $\mathrm{a}_{2}=900$ \\
4 & $\theta_{4}$ & 90 & $\mathrm{a}_{3}$ & $\mathrm{~d}_{4}$ & \pm 0 \\
5 & $\theta_{5}$ & -90 & 0 & 0 & \pm 360 & $\mathrm{a}_{3}=160$ \\
6 & $\theta_{6}$ & 90 & 0 & $\mathrm{~d}_{6}$ & \pm 115 & $\mathrm{~d}_{4}=1010.5$ \\
$\mathrm{~d}_{6}=534.5$ & \pm 450 & \\
\hline
\end{tabular}




\section{Modeling of Nickel Anode Plate Handling Robot Dynamics}

\section{Establishment of Lagrange's Equation}

There are various methods to solve the kinetic equations of the nickel anode plate handling robot, while the Lagrangian analysis method is based only on the energy term and enables the derivation of the kinetic equations in a simpler form. For the complex configuration of the designed nickel anode plate handling robot, the Lagrangian method is used to analyze the robot dynamics (Cui, 2013; Li and Zhao, 2008).

Let the turning angle variables of the six joints of the robot be:

$$
\theta=\left[\theta_{1} \theta_{2} \theta_{3} \theta_{4} \theta_{5} \theta_{6}\right]
$$

Let the driving torque of each robotic arm joint be:

$$
\mathrm{T}=\left[\mathrm{T}_{1} \mathrm{~T}_{2} \mathrm{~T}_{3} \mathrm{~T}_{4} \mathrm{~T}_{5} \mathrm{~T}_{6}\right]
$$

The kinetic energy of the robot linkage is:

$K_{\mathrm{i}}=\frac{1}{2} \sum_{i=1}^{n} \sum_{j=1}^{i} \sum_{k=1}^{i} \operatorname{Trace}\left(U_{i j} J_{i} U_{i k}^{T}\right) \dot{\theta}_{j} \dot{\theta}_{k}+\frac{1}{2} \sum_{i=1}^{n} I_{i} \dot{\theta}_{i}^{2}$

where: $U_{i j}, U_{i k}$ - derivatives of the transformation matrix with respect to the joint rotation angle.

$J_{i}$ and $I_{i}$ - are the inertia matrix and the rotational inertia of the actuator.

Since the potential energy of the robot connecting rod is the sum of the potential energy of all the rods, the potential energy of the connecting rod can be expressed as:

$$
P=\sum_{i=1}^{n} \mathrm{p}_{\mathrm{i}}=\sum_{i=1}^{n}\left[-m_{i} g^{T} \cdot{ }^{0} T_{i} \bar{r}_{i}\right]
$$

where the matrix of is composed of the projections of the gravitational acceleration of the connecting rod in the $\mathrm{x}, \mathrm{y}$ and $\mathrm{z}$ directions:

$0_{T_{i}}=$ The coordinate transformation of the coordinate system $I$ with respect to the base coordinate system

$=$ The position of the center of mass of the connecting rod in the coordinate system $i$

where the matrix of $g^{T}$ is composed of the projections of the gravitational acceleration of the connecting rod in the $x, y$ and $z$ directions.

Combined with what was stated above, the Lagrange equation can be expressed as:

$$
\begin{aligned}
& L=K-P=\frac{1}{2} \sum_{i=1}^{n} \sum_{j=1}^{i} \sum_{k=1}^{i} \operatorname{Trace}\left(U_{i j} J_{i} U_{i k}^{T}\right) \dot{\theta}_{j} \dot{\theta}_{k} \\
& +\frac{1}{2} \sum_{i=1}^{n} I_{i} \dot{\theta}_{i}^{2}-\sum_{i=1}^{n}\left[-m_{i} g^{T} \cdot{ }^{0} T_{i} \bar{r}_{i}\right]
\end{aligned}
$$

\section{Establishment of Kinetic Equations for Nickel Anode Plate Handling Robot}

The driving moment equation of each robot arm joint of the nickel anode plate handling robot can be expressed as:

$\tau_{i}=\frac{d}{d t}\left(\frac{\partial L}{\partial \dot{q}_{i}}\right)-\frac{\partial L}{\partial q_{i}} i=1,2,3,4,5,6$

$\frac{\partial L}{\partial \dot{q}_{p}}=\frac{1}{2} \sum_{i=1}^{6} \sum_{k=1}^{i} t r\left(\frac{\partial T i}{\partial q_{p}} I i \frac{\partial T i^{T}}{\partial q_{k}}\right) \dot{q}$

Each derivative term of $E_{q}$ :

$\frac{d}{d t}\left(\frac{\partial L}{\partial \dot{q}_{p}}\right)=\sum_{i=p}^{6} \sum_{k=1}^{i} t r\left(\frac{\partial T i}{\partial q_{p}} I i \frac{\partial T i^{T}}{\partial q_{k}}\right) \ddot{q_{k}}$

$+2 \sum_{i=p}^{6} \sum_{j=1}^{i} \sum_{k=1}^{i} \operatorname{tr}\left(\frac{\partial^{2} T_{i}}{\partial q_{j} \partial q_{k}} I i \frac{\partial T i^{T}}{\partial q_{k}}\right) \ddot{q}_{j} \ddot{q}_{k}$

$\frac{\partial L}{\partial \dot{q}_{p}}=\sum_{i=p}^{6} \sum_{j=1}^{i} \sum_{k=1}^{i} t r\left(\frac{\partial^{2} T_{i}}{\partial q_{p} \partial q_{j}} I i \frac{\partial T i^{T}}{\partial q_{k}}\right) \dot{q_{j}} \dot{q}_{k}+\sum_{i=p}^{6} m_{i} g^{T} \frac{\partial T_{i}}{\partial q_{p}} r_{i}$

The collation is shown below:

$$
\tau_{i}=M(q) \ddot{q}+C(q, \dot{q}) \dot{q}+G(q)
$$

\section{Nickel Anode Plate Handling Robot Dynamics Simulation}

ADAMS is used to simulate and analyze the motion of the nickel-anode plate handling robot and the $3 \mathrm{D}$ model of the nickel-anode plate handling robot is created using SolidWorks software. The overall simulation model of the nickel-anode plate handling robot is shown in Fig. 3.

\section{Establishment of the Simulation Scheme}

Firstly, define the material information for the robot model imported into ADAMS; then add constraints at the rotating joints respectively and add fixed subsets 
between the base and the ground; secondly, set the drives for the rotating subsets respectively according to the motion requirements of the robot and edit the drive functions all as follows:

1) step (time,5,0,7, -90d) +step (time, 8,0,10,11d) +step (time, 10,0,12,79d)

2) step (time, $2.5,0,4,-20 \mathrm{~d})+$ step (time, 6, 0,7,20d)

3) step (time, 7,0,8, -17.9d) +step (time, 10,0,12,17.9d)

4) 4step (time, $0,0,2,90 \mathrm{~d}$ ) +step (time, 2.5,0,4, -90d) +step (time,7,0,8, -17.9d) +step (time, 8,0,10,90d) +step (time, 10,0,12, -72.1d)

5) 0

6) Step (time,7,0,8,90d) +step (time, 10,0,12, -90d)

Marker points are added at the center of each joint for simulation and then the kinematic parameters of the Marker points on each joint are extracted and the postprocessing tools of ADAMS are used to derive the variation curves of torque, angle, angular velocity and angular acceleration of each joint of the robot over time.

\section{Adams-Based Dynamics Simulation and Analysis}

After running the simulation according to the established simulation scheme, the simulation results were obtained as shown in Fig. 4 to 10.

The angular displacement variation curve in Fig. 4 shows that the curve of spline interpolation is smoother and smoother and the movement ends with each joint returning to the initial position without error. The velocity variation curve of Fig. 5 shows that the curve of cubic polynomial interpolation is smoother, but the maximum velocity of spline interpolation is smaller while satisfying the working need. The angular velocity change curve in Fig. 6 shows that the cubic polynomial is not only less smooth and continuous than the spline interpolation, but also the maximum values of angular velocity and angular acceleration are larger than the spline interpolation. The change of angular acceleration in Fig. 7 is curvilinear, while that of the cubic polynomial is linear, which indicates that the motion of the sample interpolation is smoother. The force curve in the z-direction in Fig. 8 is gentler compared with the cubic polynomial. The cubic polynomial is more intuitive to observe that the robot is almost in the same position from $2 \mathrm{~s}$ to $4 \mathrm{~s}$ and there is almost no up and down movement, which requires high rigidity of the robot and the sample interpolation process is easier to achieve. The curve change of spline interpolation is more moderate and the maximum moment is smaller than cubic polynomial interpolation. The curve changes of both methods are more gentle. Both return from zero kinetic energy to zero kinetic energy; both can satisfy the motion process, but the maximum kinetic energy of Fig. 10 is in the base frame, which is less harmful to the body of the nickel anode plate handling robot Table 2 .

Table 2: Constraint relationships between the components of the nickel anode plate handling robot

\begin{tabular}{llll}
\hline Constraint type & $\begin{array}{l}\text { Constraint } \\
\text { name }\end{array}$ & $\begin{array}{l}\text { First building } \\
\text { block }\end{array}$ & $\begin{array}{l}\text { Second building } \\
\text { block }\end{array}$ \\
\hline Fixed joint & JOINT_1 & Base & Ground \\
Fixed joint & JOINT_2 & Clamps & Six-Axis \\
Rotating joint & JOINT_3 & Axis 1 & Base \\
Rotating joint & JOINT_4 & Axis 2 & Axis 1 \\
Rotating joint & JOINT_5 & Axis 3 & Axis 2 \\
Rotating joint & JOINT_6 & Axis 4 & Axis 3 \\
Rotating joint & JOINT_7 & Axis 5 & Axis 4 \\
Rotating joint & JOINT_8 & Axis 6 & Axis 5 \\
\hline
\end{tabular}

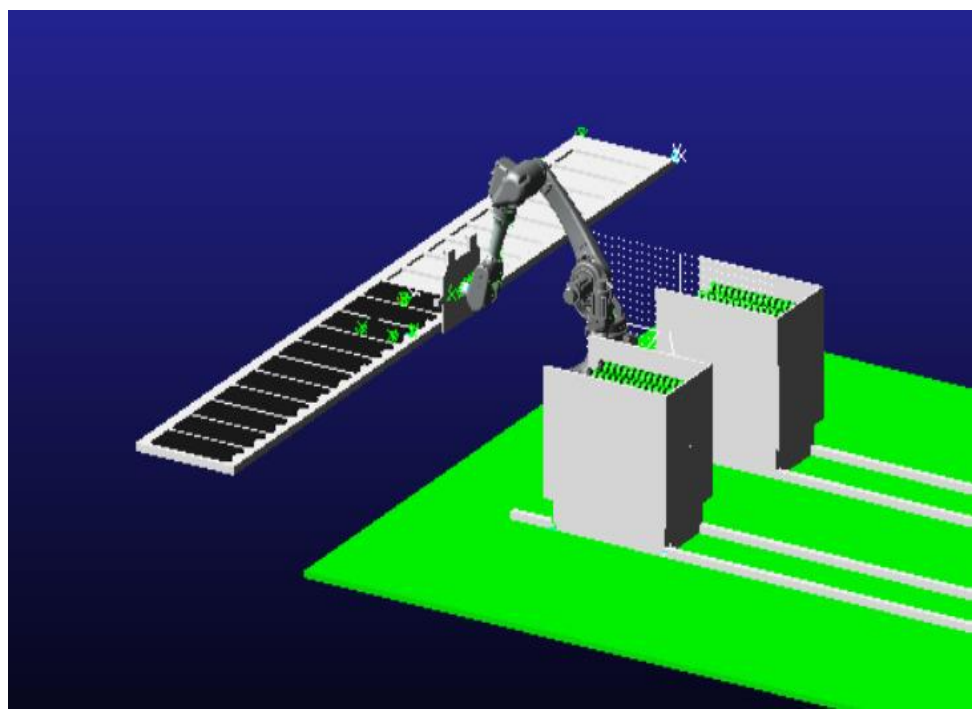

Fig. 3: Overall simulation model of nickel anode plate handling robot 
Krich Richel Mpemissi Kombo et al. / American Journal of Engineering and Applied Sciences 2021, 14 (4): 470.477 DOI: 10.3844/ajeassp.2021.470.477

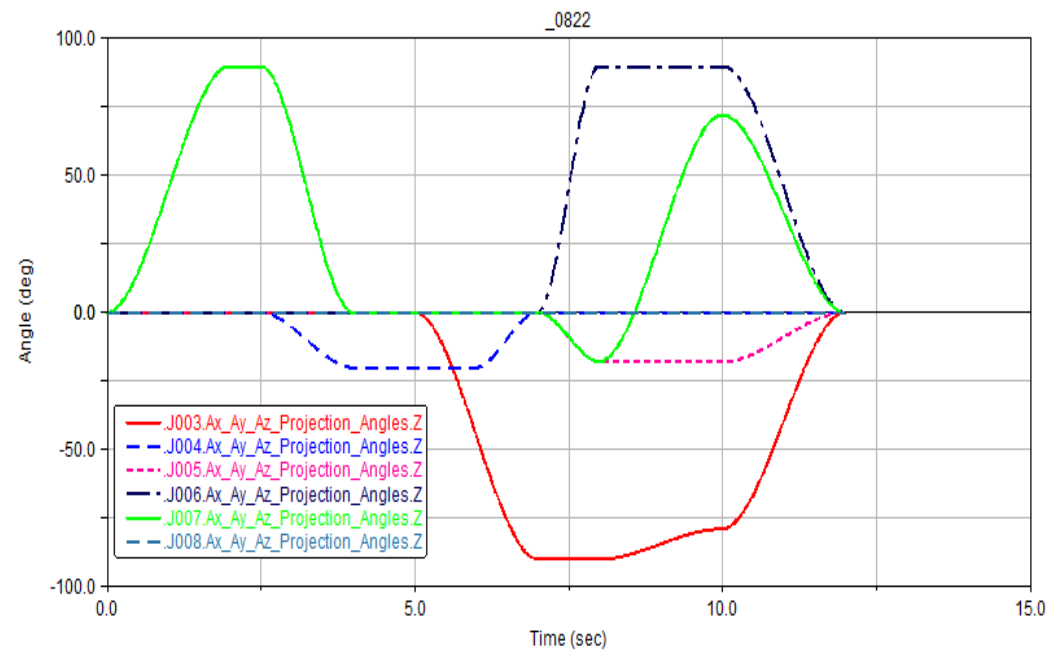

Fig. 4: Angular displacement curve of each joint

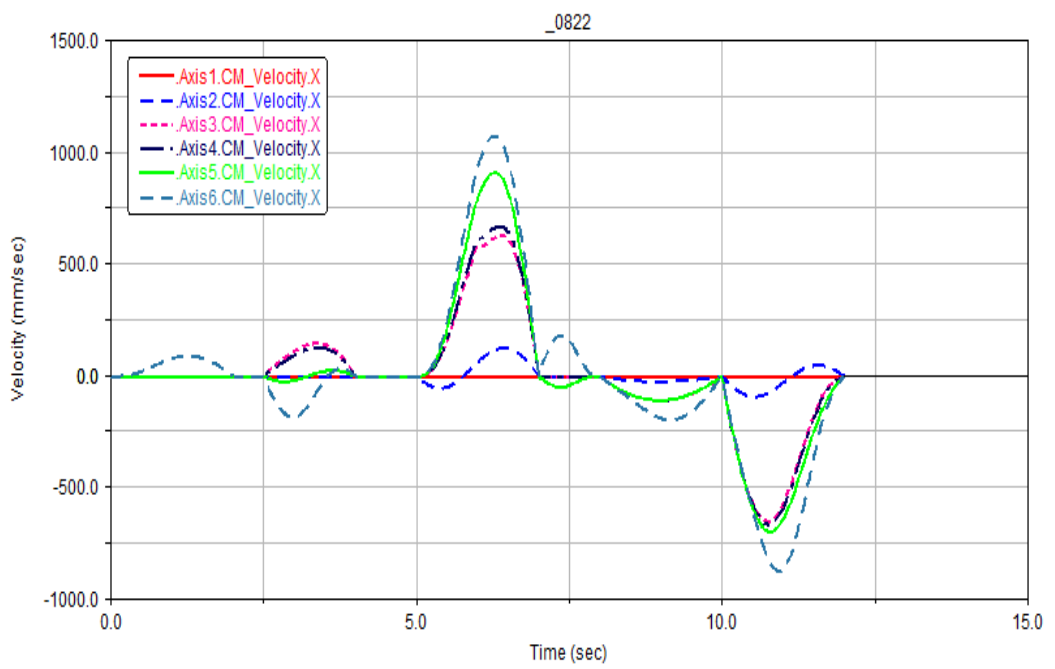

Fig. 5: Velocity profile of each joint

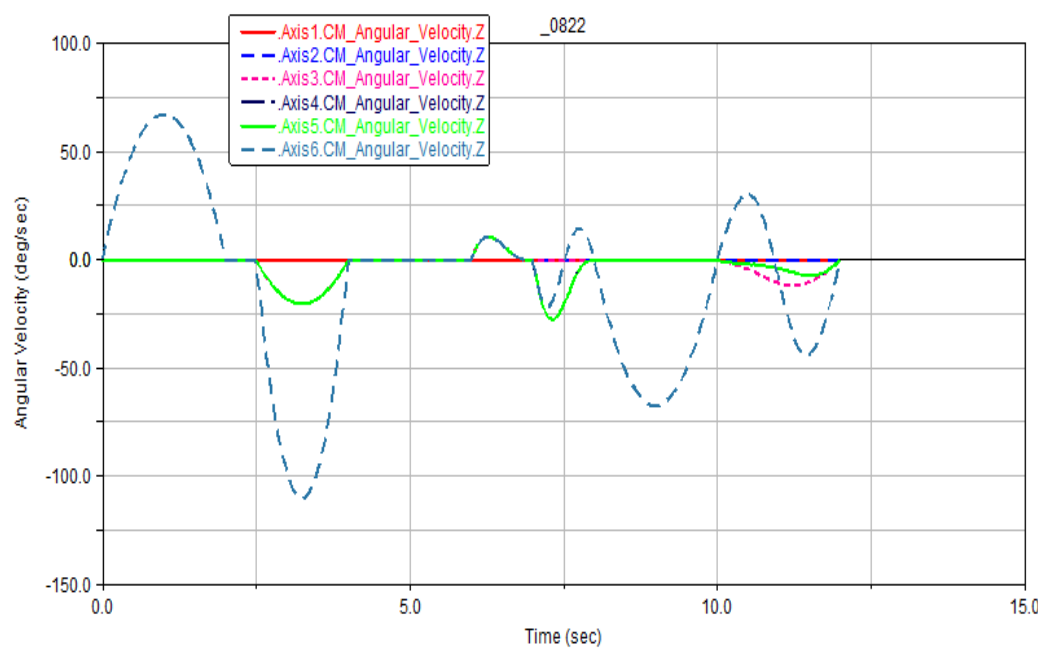

Fig. 6: Angular velocity curve of each joint 


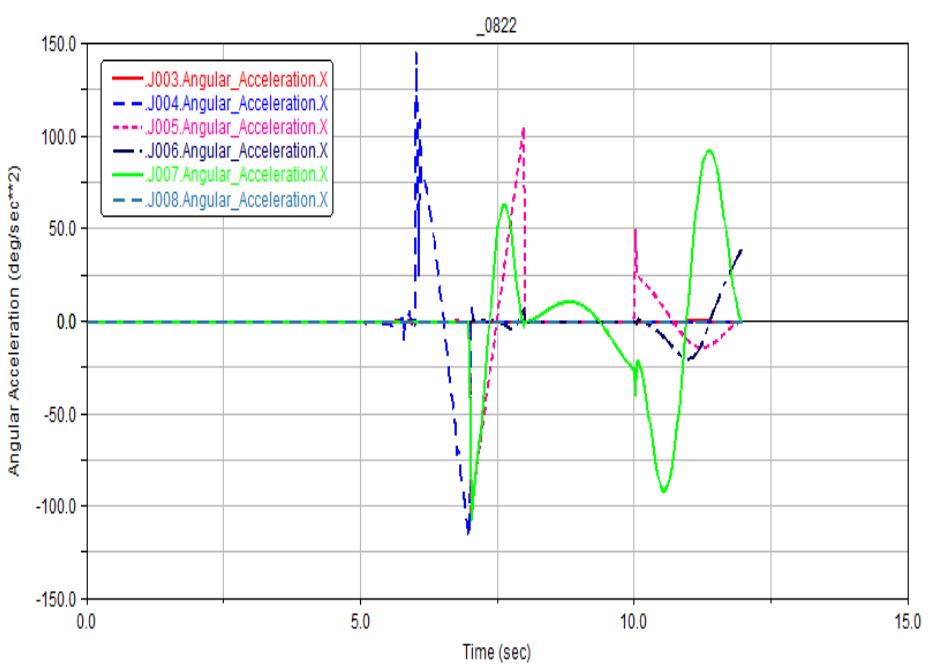

Fig. 7: Angular acceleration curve of each joint

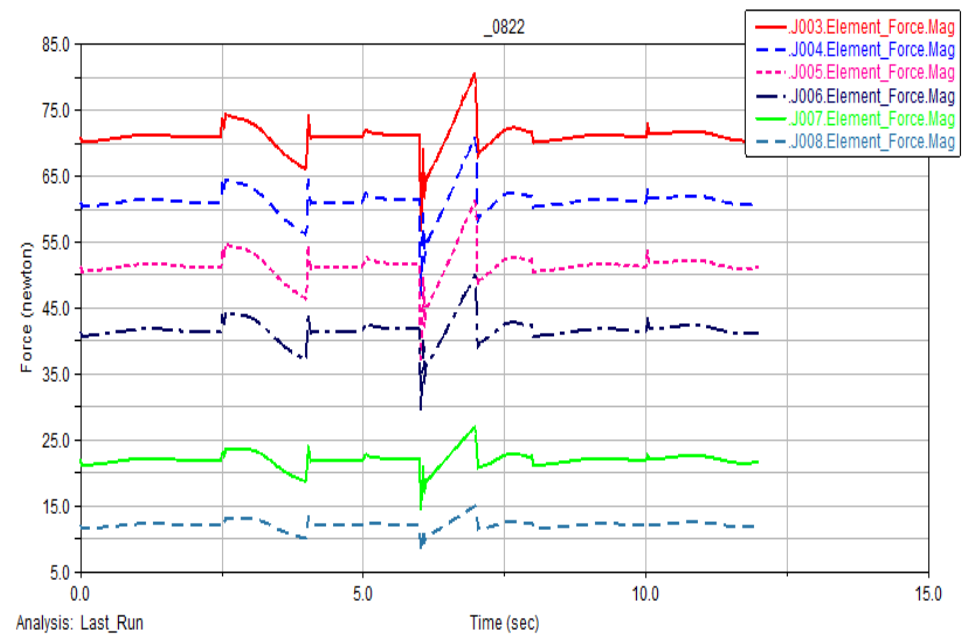

Fig. 8: Force curve of each joint in the z-direction

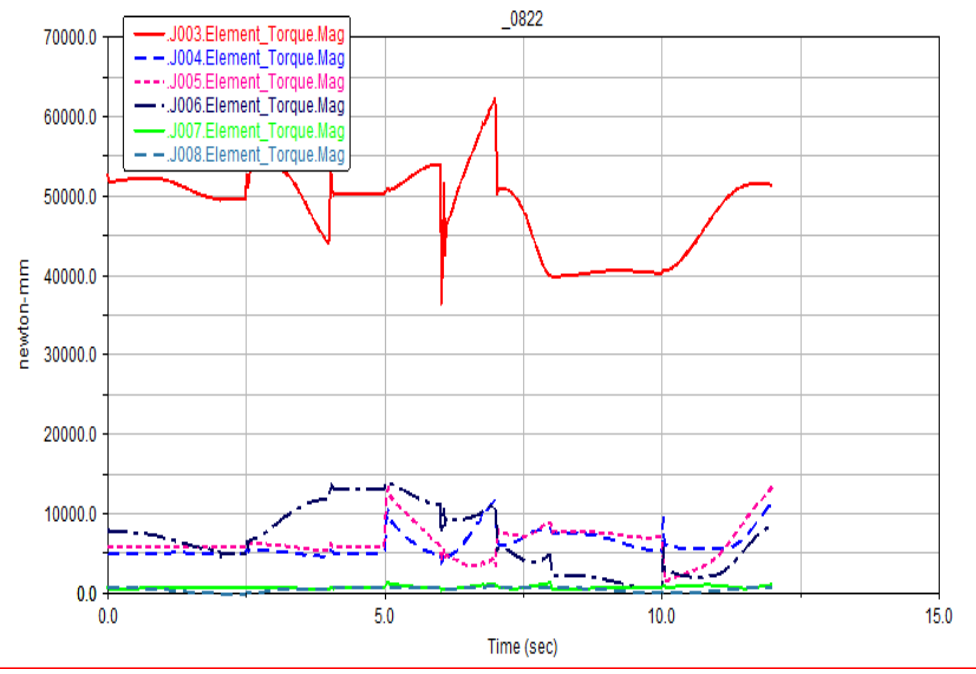

Fig. 9: Moment curve of each joint in the z-direction 


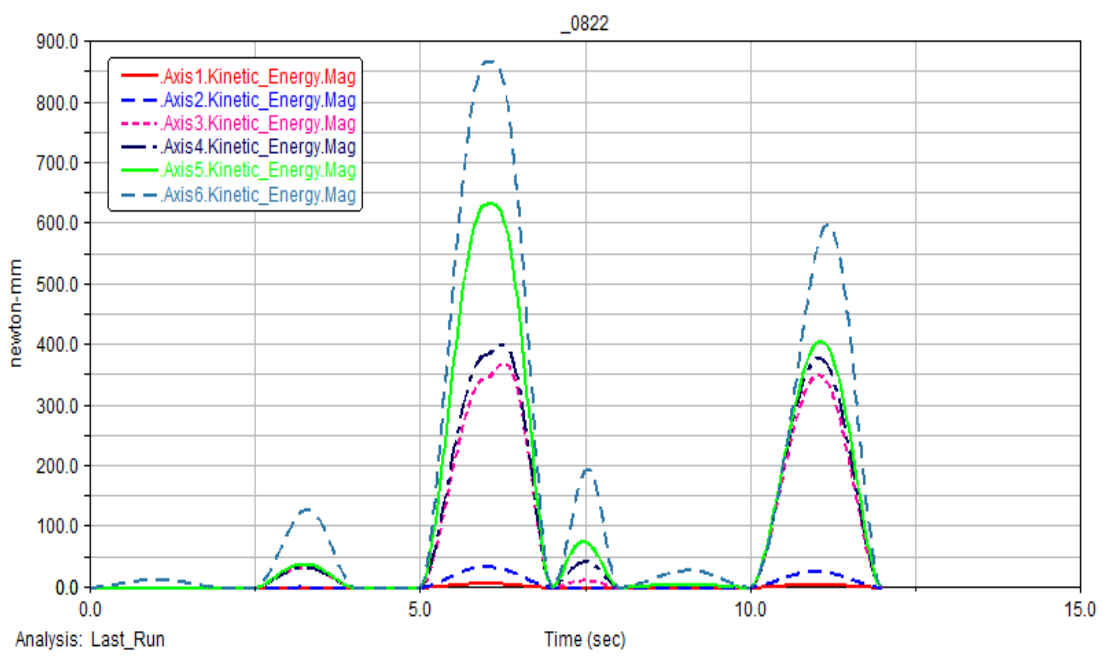

Fig. 10: Kinetic energy curve of each joint

\section{Conclusion}

Based on the three-dimensional model of the nickel-anode plate handling robot with SolidWorks software and using the D-H method to establish the linkage coordinate system and the linkage parameters, the kinetic model is solved by the Lagrangian method in this study. Based on the three-dimensional model of the nickel-anode plate handling robot, the dynamics of the robot is simulated using ADAMS software and the driving torque of each joint and the motion of the nickel-anode plate are obtained in this study. Adams dynamic simulation is of great significance to the practical application of the handling robot. The handling robot solves the problem of difficult handling of heavy objects and harmful substances with certain specifications in actual production and improves the production efficiency.

\section{Acknowledgement}

This text was acknowledged and appreciated by Dr. Guoping Li PhD supervisor in Mechanical Engineering of the University of Jinan.

\section{Author's Contributions}

Krich Richel Mpemissi Kombo: Participated in the design of the handling robot, in all the experiments, coordinated the data analysis and contributed to the major writing of the manuscript.

Guoping Li: Assisted in the designing of the research plan and organized the study, simulation and document verification.

Smart Valentine Mudzingwa: Participated in correction of the document.

\section{Ethics}

This article is an original research paper. There are no ethical issues that may arise after the publication of this manuscript.

\section{References}

Cui, M. Q. (2013). "Dynamical Modeling of SCARA Robot Based on Lagrange Formulation," 76-78. doi.org/10.19356/j.cnki.1001-3997.2013.12.024

Hu, J., Zhang, Y. P., Wang, F., Che, J. G., \& Lin, W. (2016). Kinematics simulation analysis and practical application of a handling robot $[\mathrm{J}]$. Machine Tool \& Hydraulics， 2016,44(11), 45-49. doi.org/10. 3969/j.issn.1001-3881.2016.11.011

Li, Q. L., Zhao, Y. S. (2008). Dynamics analysis and simulation of six degree of freedom industrial robot [M]. Journal of Shanghai Dian Ji University, 2008 (12).

Liu, J. I., \& Hu, R. X. (2013). ADAMS 2012 Virtual prototype from entry to master $[\mathrm{M}]$. Beijing: China Machine Press, 2013, 215-231. ISBN-10: 9787111425137.

Wang, D. J., Guan, S. Y., Chen, Y., \& Liu, Z. M. (2017). Dynamic analysis and simulation of a six-degree-offreedom handling robot $[\mathrm{J}]$. Machinery Design \& Manufacture, 2017(01), 25-29. doi.org/10.19356/j.cnki.1001-3997.2017.01.007

$\mathrm{Xu}, \mathrm{F}$. (2007). Current situation and development of industrial robot industry $[\mathrm{J}]$. Robot Technology and Application, (05), 2-4. doi.org/10.3969/j.issn.10046437.2007.05.001

Yi, Y., \& SONG, X. P. (2009). The Present Situation and Developing Trend of Robot Simulation Research [J]. Mechanical Engineer, 7, 63-65. doi.org/10.3969/j.issn.1002-2333.2009.07.031 
Krich Richel Mpemissi Kombo et al. / American Journal of Engineering and Applied Sciences 2021, 14 (4): 470.477 DOI: 10.3844/ajeassp.2021.470.477

Zeng, G., Li, Z. G. (2014) ADAMS Introductory Details and Examples $[\mathrm{M}]$. Beijing: National Defense Industry Press, 2014.7. 2014.7. Beijing. ISBN: 978-7-118-09373-5.
Zeng, J., Lin, Y. Z., Liao, X. P. (2010). Kinematics analysis and simulation of $6 \mathrm{R}$ spraying robot [J]. Machinery Design \& Manufacture, 2010 (6), 145-147. doi.org/10.19356/j.cnki.1001-3997.2010.06.062 